\title{
Attractors for the Magnetic Bénard Problem
}

\author{
Naoyuki Ishimura ${ }^{*}$, Masa Aki Nakamura ${ }^{2}$ \\ ${ }^{1}$ Hitotsubashi University, Kunitachi, Tokyo 186-8601, Japan. \\ 2 Nihon University, Kanda-Surugadai, Tokyo 101-8308, Japan. \\ * Corresponding author. Tel.: +81-42-580-8788; email: ishimura@econ.hit-u.ac.jp \\ Manuscript submitted November 26, 2014; accepted January 12, 2015. \\ doi: 10.17706/ijapm.2015.5.1.1-7
}

\begin{abstract}
The influence of the magnetic field on the heat convection is an important issue in various industrial productions. The mathematical formulation of such viscous magneto hydrodynamic fluid is given by the magnetic Bénard system. We here show the existence of attractors, which is finite dimensional and characterize the long time dynamics of the system. Furthermore, we estimate their dimensions in terms of the magnetic components as controlling parameters.
\end{abstract}

Key words: Magnetic Bénard system, attractors, fractal dimension.

\section{Introduction}

The effect of the magnetic field on the heat convection in a viscous and resistive fluid is an important topic for researches in various industrial situations, which include steel processing, the production of semi-conductors, and so on. A mathematical formulation of the heat convection phenomena under the presence of the magnetic field is provided by the magnetic Bénard equations [1], which is a coupled system of the magneto hydrodynamic equations [2] and the thermo hydraulics equations [3]. We refer to [4]-[6] for more details.

We here deal with the asymptotic behavior of solutions to the magnetic Bénard system. In particular, we are concerned with the attractors, which characterize the long-time dynamics of the original system and possess the finite dimensional structure. Indeed, the theory of infinite dimensional dissipative dynamical system developed so far and the analysis of the asymptotic behavior of solutions to the nonlinear evolution equations of these types generally show the finite dimensional features of the problems. We refer to [2], [7]-[11] for related results.

Our intention is then to estimate the fractal dimension of the attractors in terms of the various physical constants; that is, the (magnetic) Prandtl number, Rayleigh number to name a few. We believe that such characteristics may reveal the degree and the extent of the influence of magnetic factors and may be used as controlling parameters.

In this direction, the work of M. A. Boudourides and A. C. Nikoudes [12] should be recalled, where the estimate of attractors for the magnetic Bénard problem is also given as we do here. However, compared to their study, our basic model equation is slightly different since we take into account the Lorentz force, which is much suitable to industrial applications.

Our main results are Theorems 2.1 and 2.2 in the next section, which state that there exists a finite dimensional universal attractor for the magnetic Bénard system and its dimension is estimated. We exhibit these theorems after recalling our basic model as well as providing the functional analysis framework of 
the problem. We remark that the results themselves are similar to that of [12]. We conclude with discussions.

\section{Analysis of the Magnetic Bénard System}

\subsection{Model System}

We consider a homogeneous, viscous and electrically conducting fluid in $\boldsymbol{R}^{2}$, which is layered in the region $\left\{\boldsymbol{x}=\left(x_{1}, x_{2}\right) \in \boldsymbol{R}^{2} \mid 0<x_{2}<1\right\}$ and limited by the surfaces $\left\{x_{2}=0\right\},\left\{x_{2}=1\right\}$. The fluid layer is heated from below insuch a way that the lower plane is maintained at temperature $T_{0}$, while the upper plane is maintained at $T_{1}$, where $T_{0}$ and $T_{1}\left(T_{0}>T_{1}\right)$ are given constants. Thelayer is permeated perpendicularly by uniformly impressed magnetic field $\boldsymbol{H}_{\mathbf{0}}$.

In the Boussinesq approximation, the velocity $\boldsymbol{u}=\left(u_{1}, u_{2}\right)$, the pressure $p$, andthe temperature $\theta$ of the fluid, as well as the magnetic field $\boldsymbol{h}=\left(h_{1}, h_{2}\right)$ satisfythe following system of partial differential equations, which is called the magneticBénard system and will be referred to as MBS hereafter.

$$
\begin{gathered}
\frac{\partial \boldsymbol{u}}{\partial t}+(\boldsymbol{u} \cdot \nabla) \boldsymbol{u}-P_{m}(\boldsymbol{h} \cdot \nabla) \boldsymbol{h}+\frac{1}{2} P_{m} \nabla|\boldsymbol{h}|^{2}=\Delta \boldsymbol{u}+R \theta \boldsymbol{e}+Q\left(\frac{\partial \boldsymbol{h}}{\partial x_{2}}+\nabla h_{2}\right), \\
P_{m}\left(\frac{\partial \boldsymbol{h}}{\partial t}+(\boldsymbol{u} \cdot \nabla) \boldsymbol{h}-(\boldsymbol{h} \cdot \nabla) \boldsymbol{u}\right)=-\operatorname{rot} \operatorname{rot} \boldsymbol{h}+Q \frac{\partial \boldsymbol{u}}{\partial x_{2}}, \\
P_{r}\left(\frac{\partial \theta}{\partial t}+(\boldsymbol{u} \cdot \nabla) \theta\right)=\Delta \theta+R u_{2}, \\
\operatorname{div} \boldsymbol{u}=0, \quad \operatorname{div} \boldsymbol{h}=0,
\end{gathered}
$$

where, $R=\sqrt{g \alpha \beta d^{4} / \kappa v}$ denotes the square root of the Rayleigh number, $Q=\sqrt{\mu H^{2} d^{2} / 4 \pi \rho v \eta}$ the square root of the Chandrasekhar number, $P_{r}=v / \kappa$ the Prandtl number, and $P_{m}=\mu / \eta$ the magnetic Prandtl number.

Here, $g$ denotes the gravitational acceleration, $\alpha$ the coefficient of volume expansion, $\kappa$ the thermal diffusivity coefficient, $v$ the kinematic viscosity, $\mu$ the magnetic permeability, $\rho$ the density of the fluid, $\eta$ the resistivity, $d$ the depth of the layer, and $\beta=d^{-1}\left(T_{0}-T_{1}\right)$ is the temperature gradient. The unit vector $\boldsymbol{e}$ is in the $x_{2}$ direction. We note that the MBS is a perturbation system of the equilibrium state.

For the derivation of MBS, we refer to Appendix of [4]. The additional gradient terms in the equation for the velocity field $\boldsymbol{u}$ comes from the Lorentz force effect, which is important in industrial applications.

The MBS is considered with the next boundary conditions: At $x_{2}=0$ and 1 ,

$$
\boldsymbol{u}(\boldsymbol{x}, t)=\mathbf{0}, \quad h_{2}(\boldsymbol{x}, t)=\frac{\partial h_{1}}{\partial x_{2}}(\boldsymbol{x}, t)=0, \quad \theta(\boldsymbol{x}, t)=0 .
$$

It is also imposed the periodic boundary conditions:

$p, \boldsymbol{u}, \boldsymbol{h}, \theta$ and their derivatives are all periodic with period $L$ in the $x_{1}$-direction.

The initial conditions are

$$
\boldsymbol{u}(\boldsymbol{x}, 0)=\boldsymbol{u}_{\mathbf{0}}(\boldsymbol{x}), \quad \boldsymbol{h}(\boldsymbol{x}, 0)=\boldsymbol{h}_{0}(\boldsymbol{x}), \quad \theta(\boldsymbol{x}, 0)=\theta_{0}(\boldsymbol{x})
$$

on $\boldsymbol{x} \in \Omega:=(0, L) \times(0,1)$, where $\boldsymbol{u}_{\mathbf{0}}, \boldsymbol{h}_{0}, \theta_{0}$ are given functions. 
The reason why we treat the two dimensional case is that without further assumptions the global strong solution is not expected in the three dimensional case.

\subsection{Existence of Solutions}

We first settle on the functional setting. Let $\Omega:=(0, L) \times(0,1)$ as before and put $\Gamma_{1}:=\left\{x_{1}=0, L\right\}$. Define

$$
\begin{gathered}
L_{\sigma}^{2}:=\left\{\boldsymbol{u} \in\left(L^{2}(\Omega)\right)^{2} \mid \operatorname{div} \boldsymbol{u}=0 \operatorname{in} \Omega, \int_{\Omega} u_{1} d x=0, \boldsymbol{u} \text { is periodic in } x_{1}\right\}, \\
H_{\sigma}^{1}:=\left\{\boldsymbol{u} \in\left(H^{1}(\Omega)\right)^{2} \cap L_{\sigma}^{2} \mid \boldsymbol{u} \cdot \boldsymbol{n}=0 \text { on } \partial \Omega \backslash \Gamma_{1}\right\}, \\
H_{0, \sigma}^{1}:=\left\{\boldsymbol{u} \in\left(H^{1}(\Omega)\right)^{2} \cap L_{\sigma}^{2} \mid \boldsymbol{u}=0 \text { on } \partial \Omega \backslash \Gamma_{1}\right\},
\end{gathered}
$$

where $\boldsymbol{n}$ denotes the unit normal to $\partial \Omega$.

We then define

$$
H:=L_{\sigma}^{2} \times L_{\sigma}^{2} \times L^{2}(\Omega), \quad V:=H_{0, \sigma}^{1} \times H_{\sigma}^{1} \times H_{0}^{1}(\Omega),
$$

and, for $\boldsymbol{H}^{2}(\Omega)=\left\{\boldsymbol{u} \in\left(H^{2}(\Omega)\right)^{2}\right\}$,

$$
D:=\left(\left(\boldsymbol{H}^{2}(\Omega)\right)^{2} \times H^{2}(\Omega)\right) \cap V
$$

The spaces $H$ and $V$ are equipped with the scalar product and the norm respectively as follows. For

$$
\begin{gathered}
\Phi=(\boldsymbol{u}, \boldsymbol{h}, \theta), \Psi=(\boldsymbol{v}, \boldsymbol{k}, \varsigma) \in H \\
(\Phi, \Psi)=(\boldsymbol{u}, \boldsymbol{v})+(\boldsymbol{h}, \boldsymbol{k})+(\theta, \varsigma), \quad|\Phi|^{2}=(\Phi, \Phi), \\
(\Phi, \Psi)_{1}=(\boldsymbol{u}, \boldsymbol{v})+P_{m}(\boldsymbol{h}, \boldsymbol{k})+P_{r}(\theta, \varsigma), \quad|\Phi|_{1}^{2}=(\Phi, \Phi)_{1},
\end{gathered}
$$

where $(\cdot$,$) denotes the usual L^{2}$ product. For $\Phi, \Psi \in V$,

$$
\begin{gathered}
((\Phi, \Psi))=(\boldsymbol{\nabla u}, \boldsymbol{\nabla v})+(\operatorname{rot} \boldsymbol{h}, \operatorname{rot} \boldsymbol{k})+(\nabla \theta, \nabla \varsigma), \quad\|\Phi\|^{2}=((\Phi, \Phi)), \\
((\Phi, \Psi))_{1}=(\boldsymbol{\nabla u}, \boldsymbol{\nabla} \boldsymbol{v})+P_{m}(\operatorname{rot} \boldsymbol{h}, \operatorname{rot} \boldsymbol{k})+P_{r}(\nabla \theta, \nabla \varsigma), \quad\|\Phi\|_{1}^{2}=((\Phi, \Phi))_{1}
\end{gathered}
$$

Now we put

$$
b_{1}(\boldsymbol{u}, \boldsymbol{v}, \boldsymbol{w}):=((\boldsymbol{u} \cdot \boldsymbol{\nabla}) \boldsymbol{v}, \boldsymbol{w}), \quad b_{2}(\boldsymbol{u}, \theta, \varsigma):=((\boldsymbol{u} \cdot \boldsymbol{\nabla}) \theta, \varsigma)
$$

and define a continuous trili near form $B$ on $V \times V \times V$ by

$$
B\left(\Phi_{1}, \Phi_{2}, \Phi_{3}\right)=b_{1}\left(\boldsymbol{u}_{\mathbf{1}}, \boldsymbol{u}_{\mathbf{2}}, \boldsymbol{u}_{\mathbf{3}}\right)-P_{m} b_{1}\left(\boldsymbol{h}_{\mathbf{1}}, \boldsymbol{h}_{\mathbf{2}}, \boldsymbol{u}_{\mathbf{3}}\right)+P_{m} b_{1}\left(\boldsymbol{u}_{\mathbf{1}}, \boldsymbol{h}_{\mathbf{2}}, \boldsymbol{h}_{\mathbf{3}}\right)-P_{r} b_{1}\left(\boldsymbol{h}_{\mathbf{1}}, \boldsymbol{u}_{\mathbf{2}}, \boldsymbol{h}_{\mathbf{3}}\right)+P_{r} b_{2}\left(\boldsymbol{u}_{\mathbf{1}}, \theta_{2}, \theta_{3}\right)
$$


The weak formulation of the MBS is then expressed as follows.

Weak formulation of the MBS. Let $\Phi_{0}=\left(\boldsymbol{u}_{0}, \boldsymbol{h}_{0}, \theta_{0}\right) \in H$ be given. Find $\Phi=(\boldsymbol{u}, \boldsymbol{h}, \theta) \in L^{2}(0, T ; V)$ which satisfies $\Phi(0)=\Phi_{0}$ and

$$
\frac{d}{d t}(\Phi, \Psi)_{1}+((\Phi, \Psi))+B(\Phi, \Phi, \Psi)-R\left\langle M_{1} \Phi, \Psi\right\rangle-Q\left\langle M_{2} \Phi, \Psi\right\rangle=0
$$

for all $\Psi=(\boldsymbol{v}, \boldsymbol{k}, \varsigma) \in V$, where

$$
\left\langle M_{1} \Phi, \Psi\right\rangle:=(\theta \boldsymbol{e}, \boldsymbol{v})+(\boldsymbol{u} \cdot \boldsymbol{e}, \varsigma),\left\langle M_{2} \Phi, \Psi\right\rangle:=\left(\frac{\partial \boldsymbol{h}}{\partial x_{2}}, \boldsymbol{v}\right)+\left(\frac{\partial \boldsymbol{u}}{\partial x_{2}}, \boldsymbol{k}\right)
$$

We call $\Phi \in V$ a weak solution of the MBS.

If $\Phi_{0} \in V$ and $\Phi \in L^{2}(0, T ; D) \cap L^{\infty}(0, T ; V)$, then we call $\Phi$ a strong solution.

The existence of solutions to this weak formulation is now obtained straight forwardly. We just present the results.

Existence theorem. For any initial data $\Phi_{0} \in H$ and $T>0$, there exists a weaksolution $\Phi \in L^{2}(0, T ; H) \cap L^{2}(0, T ; V)$ to the MBS. If $\Phi_{0} \in V$ and $T>0$, then thereexists a strong solution $\Phi \in L^{2}(0, T ; D) \cap L^{\infty}(0, T ; V)$.

The proof involves a series of standard a priori estimates. We refer to [4], [7], [13] for the prototype of the argument.

We write in the sequel

$$
\Phi(t)=S(t) \Phi_{0}
$$

where $\{S(t)\}_{t \geq 0}$ verifies the semigroup property and will be referred to as the semigroupof the MBS.

\subsection{Attractors and the Estimate of Dimension}

We now turn our attention to the problems of attractors. First we recall the definition of absorbing sets: A subset $A \subset H$ (resp. $V$ ) is said to be absorbing in $H$ (resp. $V$ ) for the semigroup $\{S(t)\}_{t \geq 0}$ ofthe MBS, if for every bounded subset $D \subset H\left(\right.$ resp. $V$ ), there corresponds $t_{0}=t_{0}(A)$ such that

$$
S(t) D \subset A \text { for all } t \geq t_{0}(A) \text {. }
$$

As to the MBS we deal with, it can be deduced that for any $\Phi_{0} \in H$, there exists $t_{0}=t_{0}\left(\Phi_{0}\right)$ and a constant $C$ such that

$$
|\Phi(t)|_{1}^{2} \leq C\left(1+P_{r}+P_{m}\right) R^{2} \beta^{2}|\Omega|+1
$$

for all $t \geq t_{0}$ where $|\Omega|$ denotes the area of $\Omega$. Hereafter we use the same letter $C$ for various constants. Moreover, for any $\Phi_{0} \in V$ with $\left\|\Phi_{0}\right\| \leq M$, there exists $t_{0}=t_{0}(M)$ and constant $C$ such that

$$
\begin{gathered}
\frac{1}{2} \frac{d}{d t}\|\Phi(t)\|^{2} \leq 1+C\left(1+P_{r}+P_{m}\right) P_{r}{ }^{-1} R^{2} \beta^{2}|\Omega| \\
+C\left(\max \left\{1, P_{m}{ }^{-1}\right\}+\left(1+P_{r}{ }^{2}+P_{m}{ }^{2}\right)\left(1+R^{2} \beta^{2}|\Omega|\right)\|\Phi(t)\|^{2}\right)\|\Phi(t)\|^{2},
\end{gathered}
$$

together with 


$$
\int_{t}^{t+1}\|\Phi(s)\|^{2} d s \leq C\left(R^{2} \beta^{2}|\Omega|+1\right)
$$

Taking into account of the uniform Gronwall inequality (see Lemma 3.1 of [9]), we infer that the set $U \subset V$ which is defined by

$$
U:=\left\{\Phi \in V \mid\|\Phi\| \leq \mathrm{C}\left(\max \left\{1, P_{m}{ }^{-1}\right\}+\left(1+P_{r}^{2}+P_{m}{ }^{2}\right)\left(1+R^{2} \beta^{2}|\Omega|\right)\right) \cdot \exp \left[C\left(1+R^{2} \beta^{2}|\Omega|\right)\right]\right\}
$$

is an absorbing set in $V$ for the semigroup $\{S(t)\}_{t \geq 0}$.

To proceed further, we need to prepare some terminology.

Definition. A subset $X \subset V$ is said to be invariant, if there holds for every $t \geq 0$,

$$
S(t) X=X .
$$

An invariant set $X$ is said to be attracting in $H$ (resp. $V$ ), if there exists a neighborhood $O$ of $X$ in $H$ (resp. $V$ ) such that for every $\Phi \in O$,

$$
\operatorname{dist}(S(t) \Phi, X) \rightarrow 0 \text { as } t \rightarrow \infty,
$$

where the distance is taken in $H($ resp. $V$ ).

Now we consider the $\omega$-limit set $X$ of a $V$-bounded absorbing set $U$ for the semi group $\{S(t)\}_{t \geq 0}$. Precisely stated,

$$
X:=\omega(U)=\bigcap_{s \geq 0} \mathrm{Cl}_{H}\left(\bigcup_{t \geq s} S(t) U\right)
$$

where $\mathrm{Cl}_{H}$ means the closure in $H$.

By the property of the $\omega$-limit set, $X$ is a functional invariant set in $V$ whichattracts all the trajectories, and $X$ contains all the functional invariant set in $V$ for the semigroup $\{S(t)\}_{t \geq 0}$. That is, $X$ is a universal attractor.

Since the absorbing set $U$ has been proved to exist, the existence of a universal attractor for the MBS now follows.

In summary, we have established the next theorem.

Theorem 2.1. There exists a closed bounded set $X \subset V$ which is a universal attractor for the semigroup $\{S(t)\}_{t \geq 0}$ of the MBS. The set $X$ contains all the boundedfunctional invariant sets in $V$ for $\{S(t)\}_{t \geq 0}$. Moreover, there holds the estimate

$$
\|\Phi\| \leq \mathrm{C}\left(\max \left\{1, P_{m}^{-1}\right\}+\left(1+P_{r}^{2}+P_{m}^{2}\right)\left(1+R^{2} \beta^{2}|\Omega|\right)\right) \cdot \exp \left[C\left(1+R^{2} \beta^{2}|\Omega|\right)\right]
$$

for every $\Phi \in V$, where $C$ denotes a computable constants.

Proof. We just give an idea of the proof: Given an absorbing set, the existence of a universal attractor is deduced from a typical argument. Observe Theorem 1.1 of [13]. The estimate for $\Phi$ follows from that for an absorbing set.

Concerning the universal attractor $X$ proved in Theorem 2.1, we now wish to show that $X$ has a finite 
dimensional structure, and we estimate the bound of itsfractal dimension. To do this, it suffices to follow the procedure of [9]; we derive the linearized system of MBS and the evolution of $m$-dimensional volume elements panned by the solutions for this derived system. Our achievement is read as follows.

Theorem 2.2. The Hausdorff and the fractal dimension of the universal attractor $X$ proved in Theorem 1, that is, $m=\operatorname{dim}_{H} X$ and $m^{\prime}=\operatorname{dim}_{F} X$, respectively, canbe estimated by

$$
m, m^{\prime} \sim C R|\Omega|\left(P_{r}^{-1}+\beta^{2}\right)\left(1+P_{r}+P_{m}\right),
$$

where $C$ denotes a computable constant.

Proof. We also give a sketch since the proof proceeds along the strategy described in [9]; we consider the evolution of the $m$-dimensional volume element for the linearized system of $\Phi(t)$. We then find the behavior of the uniform Lyapunov exponents, Wethen employ the next observation (see $\S 4.5$ of [13]):

If the uniform Lyapunov exponents $\mu_{j}$ associated with the maximal attractor $X$ satisfy

$$
\mu_{1}+\mu_{2}+\cdots+\mu_{j} \leq-\kappa_{1} j^{2}+\kappa_{2}
$$

for every $j=1,2, \cdots$ with some positive constants $\kappa_{1}$ and $\kappa_{2}$, then the integer $l$ which is determined by

$$
l-1<2\left(\frac{\kappa_{2}}{\kappa_{1}}\right)^{\frac{1}{2}} \leq l
$$

gives an upper bound for $\operatorname{dim}_{H} X$ and $2^{-1} \operatorname{dim}_{F} X$. In our situation, we can take

$$
\kappa_{1}=\frac{C}{|\Omega|}, \quad \kappa_{2}=C R^{2}|\Omega|\left(P_{r}^{-1}+\beta^{2}\right)\left(1+P_{r}^{2}+P_{m}^{2}\right)
$$

from where the result follows. The details are omitted.

A similar estimate can be proved in the three dimensional case provided the global strong solution is assumed. We also note that the method presented so far may be potentially applied to the determination of the attractor for certain epidemic models. See for instance [13], [14].

\section{Discussions}

We have investigated the problem related to the attractors for the magnetic Bénard system (MBS). The MBS is a complexly coupled system of partial differential equations describing viscous magneto hydrodynamic fluid flows. Despite its importance in industries such as controlling aspects of the magnetic components, the mathematic a analysis of the MBS has not been substantially performed. Compared to the attempt of [12], where the estimate of the attractor is given, we include the effect of the Lorentz force and thus we expect that our study put something forwardly to such situation.

The attractor, on the other hand, characterizes the long time dynamics of the system. It is proved that the dimension of attractors is typically finite for many dissipative infinite dimensional nonlinear dynamical systems. Here we show the existence of attractors for the MBS and estimate the fractal dimension of the attractors in terms of magnetic factors. Although the result is similar to [12], we hope that our outcomes are useful even in real industries.

\section{Acknowledgment}


The authors would like to thank anonymous referee for suggesting them related works [13], [14].

The research has been supported in part by the grants of Japan Society for Promotion of Science (JSPS) (Kakenhi, Nos. 21540117, 21540147).

\section{References}

[1] Chandrasekhar, S. (1981). Hydrodynamic and Hydromagnetic Stability. New York: Dover.

[2] Sermange, S., \& Temam, R. (1983). Some mathematical questions related to the MHD equation. Communications on Pure and Applied Mathematics, 36, 635-664.

[3] Sato, S., Sano, M., \& Sawada, Y. (1988). Bifurcation to chaos and dimensionality of attractors in an extended Rayleigh-Bénard convective system. Physics Review A, 37, 1679-1683.

[4] Nakamura, M. A. (1991). On the magnetic Bénard problem I. Journal of the Faculty of Sciences, 38, 359-393. University of Tokyo, Section IA.

[5] Nakamura, M. A. (1992). On the magnetic Bénard problem II. Journal of the Faculty of Sciences, 39, 525-540. University of Tokyo, Section IA.

[6] Nakamura, M. A. (1993). Regularity and analyticity of the solutions of the magnetic Bénard problem. Advances in Mathematical Sciences and Applications, 2, 117-137.

[7] Constantin, P., \& Foias, C. (1988). Navier-Stokes Equations. Chicago: Chicago University Press.

[8] Constantin, P., Foias, C., \& Temam, R. (1985). Attractors Representing Turbulent Flow, No. 314. Memoires American Mathematical Society, Rhode Island.

[9] Foias, C., Manley, O., \& Temam, R. (1987). Attractors for the Bénard problems: Existence and physical bounds on their fractal dimension. Nonlinear Analysis T.M.A., 11, 936-967.

[10] Imai, H., Ishimura, N., \& Nakamura, M. A. (1996). Convergence of attractors for the simplified magnetic Béeard equation. European Journal of Applied Mathematics, 7, 53-62.

[11] Temam, R. (1988). Infinite-Dimensional Dynamical Systems in Mechanics and Physics. New York: Springer.

[12] Boudourides, M. A., \& Nikoudes, A. C. (1990). The attractor of the magnetic Bénard problem. Asymptotic Analysis, 3, 133-144.

[13] Peng, X.-L., Xu, X.-J., Fu, X., \& Zhou, T. (2013). Vaccination intervention on epidemic dynamics in networks. Physical Review E., 87, 022813.

[14] Shang, Y. (2013). Modelling epidemic spread with awareness and heterogeneous transmission rates in networks. Journal of Biological Physics, 39, 489-500.

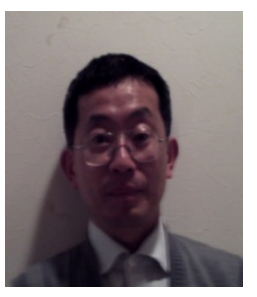

Naoyuki Ishimura graduated and obtained a PhD of mathematical sciences from the University of Tokyo, Japan. He is now a professor of mathematical finance at Graduate School of Economics, Hitotsubashi University, Tokyo, Japan. Prof Ishimura is a member of Japan Society of Industrial and Applied Mathematics (JSIAM).

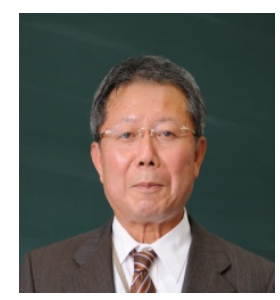

MasaAki Nakamura graduated and obtained a PhD of mathematical sciences from the University of Tokyo, Japan. He is now a professor of College of Science and Technology, Nihon University, Tokyo, Japan. Prof Nakamura is a member of Japan Society of Industrial and Applied Mathematics (JSIAM). 